\title{
ERRATUM
}

\section{Predominance of Methicillin-Resistant Staphylococcus aureus among Pathogens Causing Skin and Soft Tissue Infections in a Large Urban Jail: Risk Factors and Recurrence Rates}

\author{
Michael Z. David, Connie Mennella, Mohamed Mansour, Susan Boyle-Vavra, and Robert S. Daum \\ Section of Infectious Diseases, Department of Medicine, and Section of Infectious Diseases, Department of Pediatrics, \\ The University of Chicago, Chicago, Illinois, and Cermak Health Services, Cook County, Chicago, Illinois
}

Volume 46, no. 10, p. 3222-3227, 2008. Page 3222, abstract, line 8: "240 (63.5\%) were of MRSA and 43 (11.4\%) were of MSSA" should read "240 $(63.5 \%)$ were due to MRSA and $43(11.4 \%)$ were due to MSSA."

Page 3222, column 2, lines 10-11: "sexual activity of men who have sex with men" should read "being a man who has sex with men."

Page 3222, column 2, line 18: "may be common than recurrent MSSA SSTIs" should read "may be more common than recurrent MSSA SSTIs."

Page 3223, Table 1, column 1, row 6: "Unspecified mixed floras" should read "Unspecified mixed flora."

Pages 3224 and 3225, Table 2, subheading for column 3: "MRSA $(n=243)$ " should read "MRSA $(n=240)$."

Page 3224, Table 2, ninth row from bottom of page, column 3: "37 (19.6)" should read "47 (19.6)."

Page 3224, Table 2: The final row appearing on the page should appear four lines above its current position.

Page 3225, Table 2, footnote $t$, line 1: "HCV infection" should read "hepatitis C virus (HCV) infection." 